\title{
Accretion of gas by globular cluster stars
}

\author{
A. Thoul ${ }^{1, \star}$, A. Jorissen ${ }^{2, \star}$, S. Goriely ${ }^{2, \star}$, E. Jehin ${ }^{3}$, P. Magain ${ }^{1}$, A. Noels ${ }^{1}$, and G. Parmentier ${ }^{1}$ \\ ${ }^{1}$ Institut d'Astrophysique et de Géophysique, Université de Liège, 5 avenue de Cointe, 4000 Liège, Belgium \\ 2 Institut d'Astronomie et d'Astrophysique, CP 226, Université Libre de Bruxelles, Boulevard du Triomphe, \\ 1050 Bruxelles, Belgium \\ 3 European Southern Observatory, Casilla 19001, Santiago 19, Chile
}

Received 17 July 2001 / Accepted 7 December 2001

\begin{abstract}
Some recent observations of the abundances of $s$-process, $r$-process, and $\alpha$ elements in metal-poor stars have led to a new scenario for their formation. According to this scenario, these stars were born in a globular cluster and accreted the $s$-process enriched gas expelled by cluster stars of higher-mass, thereby modifying their surface abundances. Later on, these polluted stars evaporated from the globular cluster to constitute an important fraction of the current halo population. In addition, there are now many direct observations of abundance anomalies not only in globular cluster giant stars but also in subgiant and main-sequence stars. Accretion again provides a plausible explanation for (at least some of) these peculiarities. Here we investigate further the efficiency of the accretion scenario. We find that in concentrated clusters with large escape velocities, accretion is very efficient and can indeed lead to major modifications of the stellar surface abundances.
\end{abstract}

Key words. Galaxy: globular clusters: general - stars: abundances - stars: chemically peculiar stars: AGB and post-AGB

\section{Introduction}

Strong correlations between the $r$-process and $s$-process element abundances and the $\alpha$-element abundances in field metal-poor stars have been reported (Jehin et al. 1998, 1999), separating these stars into two sub-populations. The $r$-process elements correlate linearly with the $\alpha$ elements, with a clumping at the maximum value of $[\alpha / \mathrm{Fe}]$. The $s$-process elements, on the other hand, exhibit a more complex behavior when plotted against the $\alpha$ elements, and form a "two-branch diagram", which we show schematically in Fig. 1. We emphasize here that the observed stars, being dwarf stars, cannot have synthetized these $s$-elements in their interior.

In order to explain these observations, the EASE scenario has been developed (Jehin et al. 1999; Parmentier et al. 1999, 2000a, 2000b) that links the metal-poor field halo stars to the halo globular clusters (GCs). According to this model, the field halo stars are born in GCs. The evolution of the GCs is separated into two phases, directly connected to the two subpopulations now observed in the halo. During the first phase, "first generation" stars are formed. The initial mass function in the very

Send offprint requests to: A. Thoul, e-mail: thoul@astro.ulg.ac.be

* Chercheur Qualifié au Fonds National de la Recherche Scientifique (Belgium).

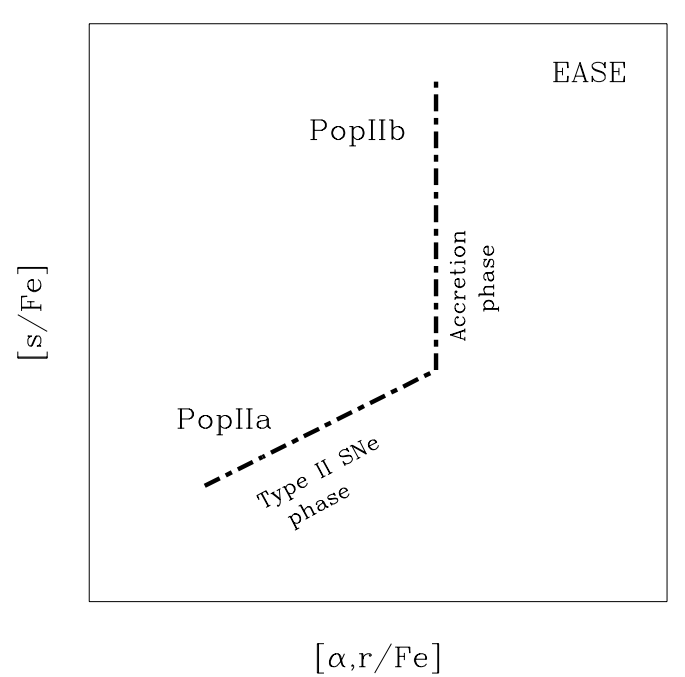

Fig. 1. The "two-branchs diagram" (see Jehin et al. 1999 for details).

metal-poor medium favors the high masses, with $3 M_{\odot}<$ $M<60 M_{\odot}$. The most massive of these stars quickly evolve to become supernovae, ejecting $\alpha$ - and $r$-process elements into the surrounding intracluster medium. The explosions trigger the formation of an outwardly expanding supershell. The primordial ISM gas is swept into the shell with the supernovae ejecta, and the resulting gas is 
enriched in $\alpha$ - and $r$-process elements. A new burst of star formation occurs in the shell, where the density is very high. This triggered star formation presumably has a Salpeter-like initial mass function. A proto-globular cluster is born. If the gravitational potential is too weak, it gets disrupted before having the chance to form a GC, and the stars become field halo stars of the PopIIa, as defined in Fig. 1. The metallicity reached in the proto-GC depends on the number of supernovae that have exploded before the proto-GC gets disrupted. The $\alpha$-element abundances are fixed by that time as well as by the initial mass function (supernova progenitors of different masses produce different relative amounts of $\alpha$ elements). The proto-globular cluster may in some cases survive this supernova phase. If the stars fall back under the effect of gravity, they form a GC. Detailed dynamical calculations have been performed (Parmentier et al. 1999) which show that proto-globular clusters can indeed survive the supernova phase and metallicities such as those observed today in GCs can be achieved in this scenario. It has also been shown that the EASE scenario, with its two separate star formation events, does not contradict observational constraints such as the narrow metallicity range found in any given cluster.

During the second phase, intermediate-mass (second generation) stars evolve, becoming asymptotic giant branch (AGB) stars. Those stars produce $s$-elements, which are brought to the surface through the so-called third dredge-up occurring after the thermal pulses. The $s$-elements are then ejected into the surrounding intracluster medium by stellar winds. The intracluster gas is therefore enriched in $s$-elements, while the $\alpha$ - and $r$-process element abundances have been fixed at their highest value after all massive stars have exploded. If the cluster can retain this enriched gas, it can be accreted by the lower-mass stars which are still on the main sequence. If the accreted material does not get mixed within the entire star but only in its convective zone, which contains about $1 \%$ of the total stellar mass for stars still near the main-sequence turnoff of present GCs, then even a small amount of accretion can lead to an appreciable enrichment of the stellar atmosphere. When the star evolves towards the giant stage, its convective zone becomes much larger, and the $s$-element enrichment is diluted considerably. We point out here that some mechanisms could be at work to already trigger the dilution of the accreted matter below the convective zone of main-sequence stars (Proffitt 1989; Proffitt \& Michaud 1989), thus diminishing the impact on the surface abundances of the deep convective envelope developing in red giant stars. We come back to this issue in more detail in Sect. 2. The accreting star can be ejected out of the GC at any time, by evaporation or by tidal forces possibly leading to the total disruption of the cluster while crossing the disk. There is indeed now observational evidence that GCs lose stars: two cannon-ball stars in 47 Tuc (Meylan et al. 1991; Jorissen \& Meylan 2001; see also Capaccioli et al. 1993; Piotto et al. 1997, and references therein), and, above all, the recent observations of "tidal tails" around many GCs (Grillmair et al. 1995; Leon et al. 2000; Odenkirchen et al. 2001). Stars enhanced in $s$-process elements by accretion and then stripped from GCs form the Pop IIb halo.

The hypothesis that the gas ejected by the intermediate-mass stars in GCs can be accreted by the other (lower-mass) cluster stars has been examined before. There is strong observational and theoretical evidence that stars reaching the AGB phase lose a large amount of mass. Several studies have been devoted to the fate of this gas in clusters (Scott \& Rose 1975; Faulkner \& Freeman 1977; Vandenberg \& Faulkner 1977; Vandenberg 1978; Scott \& Durisen 1978; Faulkner 1984; Faulkner \& Coleman 1984; Smith 1996). Stellar ejecta in GCs with shallow potential wells can leave the cluster via a smooth wind-like outflow. Faulkner \& Freeman (1977) and VandenBerg \& Faulkner (1977) find steady-state time-independent flow solutions in clusters of $10^{5} M_{\odot}$. However, they show that in GCs with deep enough potential wells, the gas can accumulate into the cluster, forming a central reservoir with a radius comparable to the GC core radius. Faulkner \& Coleman (1984) show that a small number of low-velocity low-mass stars in the cluster core can in this case accrete enough matter to form $10 M_{\odot}$ black holes, which can be considered as an extreme case. All those studies looked at what happens in present-day GCs. Smith (1996) addressed the question whether such winds might have been possible within young GCs, during epochs of much higher stellar mass-loss rates. Many GCs have high enough escape velocities to retain at least some of the stellar ejecta. A substantial amount of intracluster material could have been acquired when the turn-off mass was about $5 M_{\odot}$. In less tightly-bound clusters, the stellar ejecta are lost from the cluster either stochastically or through a continuous wind. In the cases where the stellar ejecta are retained in the cluster, the gas could be accreted by other cluster stars, thereby modifying their surface composition. Several models estimating the efficiency of the accretion scenario have appeared in the literature (see e.g. D'Antona et al. 1983; Smith 1996). A quantitative model investigating the recycling of nova ejecta has been presented by Smith \& Kraft (1996). Qualitative discussions of the accretion model have been presented by Bell et al. (1981), Norris \& Da Costa (1995), and Cannon et al. (1998).

In this paper, we study the process of accretion onto GC stars from a central reservoir of gas. In particular we take into account the evolution of this process with time. Indeed the rate of mass ejection into the cluster is strongly time-dependent, as it is highest when intermediate-mass stars reach the mass-losing stage, decreasing very rapidly as the turn-off mass decreases. In Sect. 2, we examine the possible links between halo stars and GCs. We address the problem of the "missing" intracluster gas in Sect. 3. We present some observational signatures that may possibly be related to accretion by cluster stars in Sect. 4 . In Sect. 5 we present the method used to calculate the efficiency of accretion of gas by GC stars. The results are summarized in Sect. 6. Finally Sect. 7 contains the conclusions. 


\section{Unease with the EASE scenario}

Although the EASE scenario nicely explains some abundance correlations in halo stars through a sequence of events in GCs, it faces several problems.

The GC evaporation is not the only way to populate the halo. In particular, the discovery that the Sagittarius dwarf galaxy is in the process of being tidally disrupted by our Galaxy and its stars incorporated in the halo has led to a serious reconsideration of the role of mergers in the formation of the halo. Initially put forward by Searle \& Zinn (1978), in opposition to the classical rapid collapse envisaged by Eggen et al. (1962), the merger scenario has gradually gained consensus. Relics of these past mergers may be the streamers highlighted by Majewski et al. (1996) or the blue metal-poor stars highlighted by Preston et al. (1994).

If field halo stars come from globulars, they should exhibit the same pattern of abundance peculiarities as GC stars [like C-(Na, Al) anticorrelation, N-Na-Al correlation]. Hanson et al. (1998) have shown that, based on their location in the $([\mathrm{Na} / \mathrm{Fe}],[\mathrm{Mg} / \mathrm{Fe}])$ diagram, halo field and GC stars of the same metallicity are not surrogates of one another. In particular, no field halo stars seem to exhibit the large $[\mathrm{Na} / \mathrm{Fe}]$ overabundances observed in some bright giants in GCs (Pilachowski et al. 1996). Nevertheless, the scatter in $[\mathrm{Na} / \mathrm{Fe}]$ at a given metallicity observed among halo stars (Fig. 4 of Hanson et al. 1998) is certainly large enough to accomodate the 0.3 to 0.4 dex range observed in 47 Tuc main-sequence stars and attributed to the wind pollution scenario.

If the accretion scenario is at work in GCs, one would expect that the pollution levels decrease from main sequence to giant stars as the convective envelope deepens, and dilutes the material initially restricted to a thin surface layer. Several among the chemical anomalies observed in GCs and mentioned above do not exhibit this trend, however, as they remain unchanged all the way from the main sequence to the giant branch. This suggests that the accreted matter is rapidly diluted even in main sequence stars. At least two processes may be invoked to trigger the dilution of the accreted matter into the deep radiative layers of main sequence stars. A turbulent diffusion mechanism triggered by the inversion of mean molecular weight due to the higher He content of the accreted matter has been presented by Proffitt (1989) and Proffitt \& Michaud (1989) in relation with Am and Ba stars. These authors show that even a modest composition inversion (say $\Delta X=-0.001$, and correspondingly $\Delta Y=+0.001$, where $X$ and $Y$ are the hydrogen and helium mass fractions, respectively), over the outer $10^{-3} M_{\odot}$ of the star results in dilution of the added layer by a factor of 50 in $10^{8} \mathrm{y}$. The depth reached by such a mixing may be sufficient in order to prevent further dilution during the first dredge-up. This effect may explain why the level of abundance anomalies is similar in dwarf Ba stars and in giant Ba stars (North et al. 1994), and why the level of abundance anomalies does not change between dwarf and giant stars in GCs. Another cause of mixing specific to GCs is tidal mixing caused by occasional close encounters of stars in clusters as suggested by Cox (1998).

The present paper is motivated by the connection between $s$-process enhancements in field halo stars and in cluster stars that evaporated from the cluster. There is not much evidence, however, for $s$-process anomalies due to accretion in globular cluster stars, except for $\omega$ Cen (but see the discussions of Sects. 4.1 and 6) and for the metal-poor cluster M 15 where Sneden et al. (1997) reported bimodal abundance distributions for both $[\mathrm{Ba} / \mathrm{Fe}]$ and $[\mathrm{Eu} / \mathrm{Fe}]$ (with a strong correlation between $\mathrm{Ba}$ and $\mathrm{Eu})$ in the bright giants.

\section{Low level of intracluster gas}

The absence of interstellar material in GCs has been a long-standing puzzle (e.g. Roberts 1988). Freire et al. 2001 recently achieved a positive detection of $0.1 M_{\odot}$ of ionized gas in the core of 47 Tuc. But this mass is much less than the $\sim 100 M_{\odot}$ of intracluster material expected to accumulate between two successive passages of the GC through the galactic disk (Roberts 1988). Knapp et al. (1995, 1996) have recently reinvestigated this question by setting upper limits on both the dust and ionized gas contents for a number of GCs using IRAS and radio observations, respectively. Knapp et al. (1996) conclude that their "upper limits on the mass of ionized gas are, for all observed clusters, much smaller than the amount expected to have accumulated from stellar mass loss if all that gas remains in the cluster between galactic plane passages". Simple models of the equilibrium velocity distribution of the gas supposedly photoionized by post-AGB stars indicate, however, that this gas is able to escape from the cluster in the time separating two passages through the galactic plane. Taking this effect into account, the amount of gas predicted to accumulate from stellar mass loss in this time is now well below the observational upper limits. As far as the dust is concerned, Knapp et al. (1995) conclude that the upper limits are much lower than the amounts expected to have accumulated over $10^{8}$ y from stellar mass loss.

Recycling of the mass lost by the evolved stars, by the accretion scenario investigated in the present paper, offers another way (see e.g., Freire et al. 2001 for a list of some of the other possible mechanisms) to account for the apparent lack of intracluster matter.

\section{Abundance anomalies observed in globular cluster stars, and their possible relevance to the accretion scenario}

\subsection{Peculiar red stars in $\omega$ Cen}

The GC $\omega$ Cen is discussed separately as it bears several peculiarities. It is the most massive and largest GC (Harris 1996) and exhibits a large spread in metallicity $(-1.8<[\mathrm{Fe} / \mathrm{H}]<-0.8 ;$ Norris \& Da Costa 1995) with 
some spatial asymmetry (Jurcsik 1998), but the most significant peculiarity of $\omega$ Cen in the present context is undoubtedly the presence of $\mathrm{CH}, \mathrm{Ba}$ and S stars (Dickens \& Bell 1976; Lloyd Evans 1977, 1983, 1986; Vanture et al. 1994), as listed in Table 1. Mild overabundances of the heavy elements produced by the $s$-process of nucleosynthesis are observed in the peculiar red stars (PRS) of $\omega$ Cen (Table 1). With the exception of the $\mathrm{CH}$ star ROA 279, PRS in $\omega$ Cen form the tail of the general trend of increasing heavy-element abundances with increasing $[\mathrm{Fe} / \mathrm{H}]$ typical of $\omega$ Cen (Vanture et al. 1994; Smith et al. 1995; Norris \& Da Costa 1995). PRS in $\omega$ Cen should thus not be considered as exceptional, but rather as the most extreme members of a chemically-inhomogeneous population. The PRS in $\omega$ Cen generally turn out to be somewhat enriched in carbon, since the $\omega$ Cen giants studied by Norris \& DaCosta (1995) typically have [C/Fe] -0.6, as compared to $[\mathrm{C} / \mathrm{Fe}]$ values as large as +0.2 for some of the PRS listed in Table 1. The existence of such carbonenriched giants is another specific feature of $\omega$ Cen, since in $\mathrm{GCs}$ with $[\mathrm{Fe} / \mathrm{H}] \sim-1.0$, red giants within 1 mag of the red giant tip exhibit carbon depletions by factors in the range 0.3-1.0 dex instead (Briley et al. 1992, and references therein).

The PRS of $\omega$ Cen are fainter than the luminosity threshold of the thermally-pulsing AGB $\left(M_{\mathrm{v}} \sim-2.5\right)$, according to the $M=1.25 M_{\odot}, Z=0.001$ evolutionary track of Charbonnel et al. (1996, their Fig. 2). Therefore, internal nucleosynthesis on the TP-AGB cannot account for their chemical peculiarities.

Barium and $\mathrm{CH}$ stars in the field have been shown to belong systematically to binary systems (McClure \& Woodsworth 1990), with their chemical anomalies resulting from the accretion of carbon- and $s$-process-rich matter from their AGB companion that has now evolved into a white dwarf. A radial-velocity monitoring of the PRS in $\omega$ Cen has been performed by Mayor et al. (1998), and has revealed that with the exception of the classical $\mathrm{CH}$ stars ROA 55 and ROA 70, as well as possibly the S stars ROA 320 and V6, PRS in $\omega$ Cen do NOT belong to binary systems, however (Mayor et al. 1996; Table 1). A scenario specific to GCs seems thus at work to produce these PRS. One possible scenario is that those PRS were formerly member of binary systems that were disrupted by a close encounter with another cluster star. Mayor et al. (1996) estimate that systems with periods in excess of 3 to $10 \mathrm{y}$, depending on their position in the cluster, have been disrupted by tidal encounters over the lifetime of the cluster. Before being disrupted, $\mathrm{Ba}, \mathrm{S}$ or $\mathrm{CH}$ systems with such long periods may indeed have been produced by the same mass-transfer scenario as that invoked to account for the PRS observed in the field (Jorissen et al. 1998, 1999).

Norris \& DaCosta (1995) and Cannon et al. (1998) have suggested instead that the PRS envelope has been polluted by winds expelled in the intracluster medium by an early generation of AGB stars. However, the simultaneous increase of Fe and the $s$-process elements observed in $\omega$ Cen requires as well a source of Fe that cannot be delivered by AGB stars, a statement not easy to reconcile with the pollution scenario, as discussed by Smith et al. (1995). Such peculiarities in $\omega$ Cen are more in favor of a merging between two different clusters, as further discussed in Sect. 6, which also shows that accretion is not at all efficient in $\omega$ Cen.

\subsection{Abundance anomalies in main sequence or subgiant stars}

Abundance anomalies have long been known in GC giant stars (see e.g. Kraft 1994 and DaCosta 1997 for recent reviews). However, the dredge-up processes occurring in these stars make it very difficult to disentangle intrinsic from extrinsic causes to the observed chemical peculiarities. Such dredge-up processes are not expected to occur in main sequence stars, according to canonical stellar evolution theories. Abundance anomalies in those stars therefore point in principle to external causes. Because of the faintness of main sequence stars in GCs, abundance analyses of these stars have but only recently become feasible. Indications for anomalies have since then rapidly accumulated. They may be grouped into two classes - $\mathrm{CN}$ and $\mathrm{Na}$ anomalies - which are discussed in turn.

\subsubsection{CN anomalies in main sequence stars}

The bimodal nature of the distribution of the $\mathrm{CN}$ band strength of red giant stars in several GCs has been recognized since the early 1980s (see references in Kraft 1994 and Cannon et al. 1998). This property has recently been extended to main sequence stars in 47 Tuc (Bell et al. 1983; Briley et al. 1994; Cannon et al. 1998), M5 and NGC 6752 (Suntzeff \& Smith 1991), and M 13 and M 71 (Briley \& Cohen 2001). The ratio of CN-weak to CN-rich stars is similar among dwarf and more luminous stars, implying little change in the overall distribution of $\mathrm{CN}$ with evolutionary state (Briley et al. 1994). CN-strong stars tend to be more numerous in the central region of 47 Tuc (Norris \& Freeman 1979; Freeman 1985).

Recently, Grundhal et al. (1998) have suggested that the significant scatter in the Strömgren $c_{1}$ index observed along the red giant branch down to the turnoff for all clusters more metal-poor than $[\mathrm{Fe} / \mathrm{H}]=-1.2$ is attributable to star-to-star abundance variations in the CNO elements through the strength of the $\mathrm{CH}$ G band and the violet CN bands.

The anticorrelation between the $\mathrm{CN}$ and $\mathrm{CH}$ band strengths is equally found in dwarf and giant stars (e.g., Cannon et al. 1998; Briley \& Cohen 2001 and references therein), and indicates that a $\mathrm{C}$ deficiency goes along with a $\mathrm{N}$ overabundance. Contrary to internal mixing scenarios, the primordial scenarios generally invoked to account for these anomalies extending down to the main sequence are difficult to reconcile with $\mathrm{C}$ depletions. This difficulty may be circumvented if "C-poor" stars represent in fact the primordial $\mathrm{C}$ abundance level while $\mathrm{C}$-rich stars have accreted carbon, as already suggested in the case of $\omega$ Cen 
Table 1. Abundances, luminosities, and binary properties of PRS in $\omega$ Cen. Column 1 provides the ROA star number from Woolley (1966; last three digits as in Woolley's Tables I and II, or variable numbers from Woolley's Table III). Column 2 gives the spectral type from the reference mentioned in Col. 3, Cols. 4 and 5 list $M_{\mathrm{v}}$ (adopting a distance modulus of 13.92; Harris 1996) and $B-V$ from Mayor et al. 1998, Cols. 6-8 (taken from Mayor et al. 1998) give the uncertainty $\epsilon_{V_{\mathrm{r}}}$ of the mean radial velocity, the rms value $\sigma_{V_{\mathrm{r}}}$ for stars with more than one measurement and the probability $P\left(\chi^{2}\right)$ that the observed $\sigma_{V_{\mathrm{r}}}$ is due to observational error only. Column 9, labelled $V_{\mathrm{r}}$, provides a flag characterizing the binary nature of the star, according to the following rules: a star is considered as spectroscopic binary ("SB") if $P\left(\chi^{2}\right)<0.01$ and $\sigma_{V_{\mathrm{r}}}>2.0 \mathrm{~km} \mathrm{~s}$. The latter condition is imposed because red giants close to the RGB tip are known to exhibit a radial-velocity jitter of the order of $1 \mathrm{~km} \mathrm{~s} \mathrm{~s}^{-1}$ (Mayor et al. 1984; see also Fig. 1 of Jorissen et al. 1998). If $0.01 \leq P\left(\chi^{2}\right)<0.05$, or $P\left(\chi^{2}\right)<0.01$ and $\sigma_{V_{\mathrm{r}}}>1.5 \mathrm{~km} \mathrm{~s}$, the star is considered as variable ("var"). Columns $10-12$ list the $[\mathrm{Fe} / \mathrm{H}],[\mathrm{C} / \mathrm{Fe}]$ and $s$-process abundances when available, with $[s / \mathrm{Fe}]=$ $([\mathrm{Y} / \mathrm{Fe}]+[\mathrm{Zr} / \mathrm{Fe}]) / 2$. Column 13 provides the reference for the abundance data.

\begin{tabular}{|c|c|c|c|c|c|c|c|c|c|c|c|c|}
\hline $\mathrm{ROA}$ & Sp. & Ref & $M_{\mathrm{v}}$ & $B-V$ & $\epsilon_{V_{\mathrm{r}}}$ & $\sigma_{V_{\mathrm{r}}}$ & $P\left(\chi^{2}\right)$ & $V_{\mathrm{r}}$ & {$[\mathrm{Fe} / \mathrm{H}]$} & {$[\mathrm{C} / \mathrm{Fe}]$} & {$[s / \mathrm{Fe}]$} & Ref \\
\hline 55 & $\mathrm{CH}$ & 1 & -2.43 & 1.74 & 0.74 & 3.98 & 0.000 & SB & & & & \\
\hline 70 & $\mathrm{CH}$ & 1 & -2.34 & 1.82 & 0.90 & 4.33 & 0.000 & SB & & & & \\
\hline V53 & $\mathrm{Ba}$ & 2 & -1.97 & 1.78 & 0.50 & 1.00 & 0.015 & var & & & & \\
\hline 201 & $\mathrm{~S} 2.5 / 1-2$ & 3 & -1.82 & 1.61 & 0.52 & 1.04 & 0.030 & var & -0.85 & -0.30 & 0.33 & 4 \\
\hline 219 & $\mathrm{Ba}$ & 2 & -1.76 & 1.68 & 0.41 & 1.01 & 0.003 & var & -1.25 & 0.22 & 0.40 & 4 \\
\hline 231 & $\mathrm{Ba}$ & 2 & -1.73 & 1.63 & 0.40 & 1.56 & 0.000 & var & -1.10 & -0.60 & 0.44 & 4 \\
\hline 243 & $\mathrm{Ba}$ & 2 & -1.70 & 1.31 & 0.31 & 0.70 & 0.155 & & & & & \\
\hline 248 & $\mathrm{Ba}$ & 2 & -1.69 & 1.84 & 0.46 & 1.44 & 0.000 & var & -0.78 & - & 0.17 & 4 \\
\hline 270 & $\mathrm{Ba}$ & 2 & -1.61 & 1.53 & 0.37 & 0.99 & 0.014 & var & -1.24 & -0.01 & 0.34 & 4 \\
\hline 276 & $\mathrm{Ba}$ & 2 & -1.61 & 1.52 & 0.37 & 0.82 & 0.113 & & & & & \\
\hline 279 & $\mathrm{CH}$ & 1 & -1.60 & 1.48 & 0.33 & 0.88 & 0.209 & & -1.70 & - & 0.48 & 4 \\
\hline 286 & $\mathrm{Ba}$ & 2 & -1.59 & 1.57 & 0.25 & 0.45 & 0.774 & & & & & \\
\hline 287 & $\mathrm{Ba}$ & 2 & -1.59 & 1.48 & 0.30 & 0.67 & 0.335 & & -1.43 & 0.06 & 0.26 & 4 \\
\hline 300 & $\mathrm{~S} 2 / 1-2$ & 3 & -1.56 & 1.58 & 0.27 & 0.53 & 0.548 & & & & & \\
\hline 316 & $\mathrm{Ba}$ & 2 & -1.50 & 1.66 & 0.58 & 1.16 & 0.017 & var & & & & \\
\hline 320 & $\mathrm{~S} 2 / 1-2$ & 3 & -1.49 & 1.65 & 0.86 & 2.58 & 0.000 & SB? & & & & \\
\hline 321 & $\mathrm{Ba}$ & 2 & -1.48 & 1.45 & 0.23 & 0.27 & 0.945 & & & & & \\
\hline V6 & $\mathrm{S} 3 / 1$ & 3 & -1.47 & 1.54 & 1.18 & 2.35 & 0.000 & SB? & & & & \\
\hline 324 & $\mathrm{Ba}$ & 2 & -1.46 & 1.59 & 0.25 & 0.39 & 0.749 & & & & & \\
\hline 332 & $\mathrm{Ba}$ & 2 & -1.45 & 1.53 & 0.31 & 0.75 & 0.115 & & & & & \\
\hline 357 & $\mathrm{Ba}$ & 2 & -1.42 & 1.48 & 0.48 & 1.17 & 0.000 & var & -0.85 & - & 0.20 & 4 \\
\hline 371 & $\mathrm{~K} 5 \mathrm{Ba}$ & 3 & -1.38 & 1.74 & 0.51 & 1.25 & 0.000 & var & $\begin{array}{l}-0.79 \\
-1.0\end{array}$ & $\begin{array}{l}-0.46 \\
-\end{array}$ & $\begin{array}{l}0.46 \\
0.8\end{array}$ & $\begin{array}{l}4 \\
5\end{array}$ \\
\hline 421 & $\mathrm{Ba}$ & 2 & -1.25 & 1.50 & 0.35 & 0.86 & 0.035 & var & & & & \\
\hline 425 & $\mathrm{~S} 3 / 2$ & 3 & -1.24 & 1.69 & 0.33 & 0.31 & 0.890 & & & & & \\
\hline 447 & $\mathrm{~S} 2 / 2$ & 3 & -1.18 & 1.71 & 0.28 & 0.63 & 0.338 & & & & & \\
\hline 451 & $\mathrm{Ba}$ & 2 & -1.18 & 1.53 & 0.41 & 0.92 & 0.046 & var & & & & \\
\hline V17 & $\mathrm{Ba}$ & 2 & -1.17 & 1.80 & 0.65 & 1.46 & 0.000 & var & & & & \\
\hline 480 & $\mathrm{Ba}$ & 2 & -1.15 & 1.51 & 0.49 & 0.84 & 0.133 & & -0.95 & -0.65 & 0.38 & 4 \\
\hline 505 & $\mathrm{Ba}$ & 2 & -1.09 & 1.28 & 0.27 & 0.61 & 0.363 & & & & & \\
\hline 513 & $\mathrm{~S} 3 / 2$ & 3 & -1.08 & 1.67 & 0.53 & 0.92 & 0.065 & & & & & \\
\hline 577 & $\mathrm{CH}$ & 1 & -0.97 & 1.55 & 0.53 & 1.30 & 0.008 & var & & & & \\
\hline
\end{tabular}

References to spectral types and abundances:

(1) Mayor et al. (1998), and references therein; (2) Lloyd Evans (1986); (3) Lloyd Evans (1983); (4) Norris \& Da Costa (1995); (5) Vanture et al. (1994).

by Norris \& DaCosta (1995). The CN-CH anticorrelation should thus be re-interpreted in terms of accretion of either C-poor N-rich matter, or C-rich N-poor matter. In the context of the accretion scenario, this situation may result depending on whether or not hot-bottom-burning (e.g. Boothroyd et al. 1993) was operating in the polluting AGB star, as already considered in the simple model developed by Norris \& DaCosta (1995). This interpretation of the $\mathrm{CN}-\mathrm{CH}$ anticorrelation requires that ejecta from different AGB stars (being either C-poor N-rich, or C-rich $\mathrm{N}$-poor) do mot mix in the intracluster gas, a constraint difficult to reconcile however with the model of the central gas reservoir developed in Sect. 5 .

\subsubsection{Na anomalies in main sequence and subgiant stars}

Enhancements of $\mathrm{Na}$ and $\mathrm{Al}$ are common in GC giants, and are correlated with $\mathrm{N}$ enhancements, and $\mathrm{C}$ and O depletions (Cottrell \& Da Costa 1981; Norris et al. 1981; Da Costa 1997, and references therein). The first 
indication that $\mathrm{Na}$ enhancements are observed as well in main sequence stars has been provided by Briley et al. (1995) for 47 Tuc. The reported enhancements ([Na/Fe] 0.3 to $0.4 \mathrm{dex})$ in three $\mathrm{CN}$-strong main sequence stars $\left(M_{\mathrm{v}} \sim+4\right)$ are similar to those observed in evolved stars $\left(M_{\mathrm{v}} \sim-1.5\right)$ of 47 Tuc (Cottrell \& Da Costa 1981; Gratton et al. 1986; Brown \& Wallerstein 1992).

Very recent observations of turn-off and subgiant stars in GCs show that they exhibit clear O-Na and Mg-Al anticorrelations (Gratton et al. 2001), a behavior which had previously been seen only in GC giant stars. This result cannot be explained by deep mixing scenarios and requires some other mechanism, such as accretion of polluted intracluster gas (Ventura et al. 2001).

These anomalies that clearly point against an internal mixing scenario do not seem restricted to massive, metal-rich clusters like 47 Tuc, since subgiant stars observed by King (1998) in the metal-poor cluster M 92 $([\mathrm{Fe} / \mathrm{H}]=-2.5)$ also reveal large $\mathrm{Na}$ overabundances (0.8 dex) correlated with $\mathrm{Mg}$ deficiencies. Interestingly enough, these $\mathrm{Na}$ overabundances are accompanied by modest Ba overabundances (0.4 dex).

There are nevertheless indications that qualitatively different processes may be at work in metal-poor and metal-rich clusters. For example, the constancy of the ratio of $\mathrm{CN}$-weak/CN-rich stars from the main sequence to the RGB observed in 47 Tuc, along with the constancy of the $[\mathrm{Na} / \mathrm{Fe}]$ abundances, contrast with the progressive $\mathrm{Na}$ enhancements and $\mathrm{C}$ depletions with increasing luminosity reported for the more metal-poor clusters M 13 (Pilachowski et al. 1996), M 4 and NGC6752 (Suntzeff \& Smith 1991). To complicate the picture further, the opposite trend is observed in M 92, since the Na overabundances in the subgiants (King et al. 1998) are larger than in the RGB-tip giants (Shetrone 1996). It should be remarked that, in the context of the accretion scenario, a more relevant distinctive parameter might be the cluster mass (47 Tuc is indeed very massive) rather than its metallicity.

In addition, Armosky et al. (1994) has observed that in some clusters, the abundances of $\mathrm{Y}, \mathrm{Ba}, \mathrm{Ce}$, and $\mathrm{Nd}$ in giant stars are nearly constant as $\mathrm{Na} / \mathrm{Fe}$ varies widely.

The different observed behaviors in the Na abundances clearly reflects the diversity of the nucleosynthetic processes which produce that chemical element. Sodium and $s$-elements are efficiently produced through proton mixing by the NeNa cycle (see for example Figs. 1 and 7 in Goriely \& Mowlavi 2000). On the other hand, the Naproduction during the third dredge-up is not correlated to the $s$-process (Mowlavi 1999). Finally, $\mathrm{Na}$ is produced in massive stars through the combustion of carbon (Woosley \& Weaver 1995).

\subsection{Period variations of RR Lyrae in globular clusters}

Cox (1998) has suggested that the slight period decrease $\left(2.5 \times 10^{-5} \mathrm{~d}\right.$ over $\left.80 \mathrm{y}\right)$ observed for the RR Lyr V53 in
M 15, which cannot be attributed to secular evolutionary effects, is caused by the accretion of about $10^{-7} M_{\odot}$ over $\approx 100 \mathrm{y}$. The corresponding accretion rate $\left(10^{-9} M_{\odot} \mathrm{y}^{-1}\right)$ is however much larger than that predicted to result from the accretion of gas from a central reservoir in M 15. Table 3 predicts that a $1 M_{\odot}$ star accretes $0.07 M_{\odot}$ over the cluster lifetime of $\sim 10^{10} \mathrm{y}$, resulting in an average accretion rate of $7 \times 10^{-12} M_{\odot} \mathrm{y}^{-1}$.

\section{The accretion scenario}

It is worthwhile to attempt to understand the fate of all the interstellar matter ejected by massive and intermediate-mass stars during the early phases of a GC's evolution. On the one hand, it can bring an answer to the apparent lack of ISM in present-day GCs and, on the other hand, if the efficiency of the accretion process can be related to some properties of GCs, it may be expected to unravel why GCs exhibit different abundance patterns in their main-sequence, turn-off and subgiant stars.

\subsection{Bondi accretion rate}

Following Bondi's (Bondi 1952) model for spherical accretion, the rate of mass accretion by a star of mass $M_{\mathrm{s}}$ is given by

$\frac{\mathrm{d} M_{\mathrm{s}}}{\mathrm{d} t}=4 \pi \lambda\left(G M_{\mathrm{s}}\right)^{2} \rho_{\mathrm{g}}\left(v_{\text {rel }}^{2}+c_{\mathrm{s}}^{2}\right)^{-3 / 2}$

where $\lambda$ is a constant of order unity, $\rho_{\mathrm{g}}$ is the unperturbed gas density, $c_{\mathrm{s}}$ is the sound speed in the gas, and $v_{\text {rel }}$ is the relative velocity of the star with respect to the gas.

In what follows, all masses are given in units of $1 M_{\odot}$.

\subsection{Mass loss by $A G B$ stars}

First we look at the amount of gas ejected by the AGB stars. We assume that the stars eject their mass instantaneously when reaching the AGB phase. The rate of gas production by AGB stars is given by

$\frac{\mathrm{d} m_{\mathrm{g}}}{\mathrm{d} t}=-m_{\mathrm{ej}} \frac{\mathrm{d} N}{\mathrm{~d} t}$

where $m_{\mathrm{ej}}$ is the mass ejected by AGB stars, and $\mathrm{d} N / \mathrm{d} t$ is the rate at which stars reach the AGB phase. We can write

$\frac{\mathrm{d} N}{\mathrm{~d} t}=\frac{\mathrm{d} N}{\mathrm{~d} m} \frac{\mathrm{d} m}{\mathrm{~d} t}$

where $\mathrm{d} N / \mathrm{d} m$ is the initial mass function (IMF), giving the number $N$ of stars born in a given mass interval $(m, m+\mathrm{d} m)$, and $\mathrm{d} m / \mathrm{d} t$ is the rate at which stars in this mass range reach the AGB phase. We choose a simple power-law initial mass function:

$\frac{\mathrm{d} N}{\mathrm{~d} m}=K_{1} m_{\mathrm{cl}, 0} m^{-\alpha}$

where $m_{\mathrm{cl}, 0}$ is the total initial cluster mass, $\alpha$ is the powerlaw index, and the normalization constant

$K_{1}=(\alpha-2)\left(m_{1}^{2-\alpha}-m_{\mathrm{u}}^{2-\alpha}\right)^{-1}$ 
where $m_{1}$ and $m_{\mathrm{u}}$ are the lower and upper mass limits of the mass spectrum. For a Salpeter-like IMF, we have $\alpha=2.35$, whereas if we want to favor the higher mass stars we choose a flatter spectrum with, e.g., $\alpha=1.5$. The evolution time $t_{\mathrm{MS}}$ (in y) of a star of mass $m$ (between 1 and $\left.10 M_{\odot}\right)$ along the main sequence can be approximated by (Bahcall \& Piran 1983)

$\log t_{\mathrm{MS}}=10-3.6 \log m+(\log m)^{2}$.

Here we neglect the additional post-main-sequence evolution time, which is small compared to $t_{\mathrm{MS}}$. From this relation we get

$\frac{\mathrm{d} m}{\mathrm{~d} t_{\mathrm{MS}}}=\frac{m}{t_{\mathrm{MS}}} \frac{1}{2 \log m-3.6}$.

Finally, we adopt the updated results of Weidemann (2000) for the initial-to-final mass relation. We fit their results:

$m_{\mathrm{f}}= \begin{cases}0.053 m_{\mathrm{i}}+0.497 & \text { if } 1.0 \leq m_{\mathrm{i}} \leq 2.5 \\ 0.113 m_{\mathrm{i}}+0.347 & \text { if } 2.5 \leq m_{\mathrm{i}} \leq 4.0 \\ 0.073 m_{\mathrm{i}}+0.507 & \text { if } 4.0 \leq m_{\mathrm{i}} \leq 8.0\end{cases}$

where $m_{\mathrm{f}}$ and $m_{\mathrm{i}}$ are the final and initial masses of the star, so that $m_{\mathrm{ej}}=m_{\mathrm{i}}-m_{\mathrm{f}}$. Putting together Eqs. (2)-(8), we can calculate the rate at which the gas is ejected by the AGB stars as a function of time. The total amount of gas which has been ejected by the AGB stars in the cluster after a given time $t$ is obtained by integrating the gas production rate:

$m_{\mathrm{g}}(t)=\int_{t_{1}}^{t} \frac{\mathrm{d} m_{\mathrm{g}}}{\mathrm{d} t^{\prime}} \mathrm{d} t^{\prime}$,

where $t_{1}$ is the time at which the most massive stars (of mass $m_{\mathrm{u}}$ ) reach the AGB, i.e., $\log t_{1}=10 .-3.6 \log m_{\mathrm{u}}+$ $\left(\log m_{\mathrm{u}}\right)^{2}$. In Fig. 2, we show (thin solid line) $m_{\mathrm{g}}(t)$ for $m_{\mathrm{u}}=8, m_{\mathrm{l}}=0.1$, and $\alpha=2.35$. To illustrate the sensitivity on the IMF parameters, we also show the results obtained for other values of these parameters. Increasing the upper limit of the mass spectrum of course increases the number of high mass stars, resulting in more matter being ejected. Lowering the lower mass limit $m_{1}$ increases the total mass situated in low-mass stars, thus decreasing the relative number of high-mass stars. By far the most sensitive parameter is $\alpha$, the power-law index of the IMF. Choosing a flatter mass spectrum $(\alpha=1.5)$ considerably increases the number of high-mass stars in the cluster, leading to a much larger amount of gas ejected into the cluster's ISM. With this value of $\alpha$ we find that about $60 \%$ of the cluster's initial mass can be returned to the cluster as gas in $10^{10} \mathrm{y}$, whereas for a Salpeter's IMF, this number is $20 \%$. We also see that the rate of gas production in the cluster is very high at early times, and decreases very quickly as the most massive stars leave the AGB phase. Note that in this figure $m_{\mathrm{g}}$ is the total amount of matter which has been lost by the cluster's AGB stars. This is different from the amount of gas available for accretion, since some of it could have already been accreted, or could escape before being accreted.

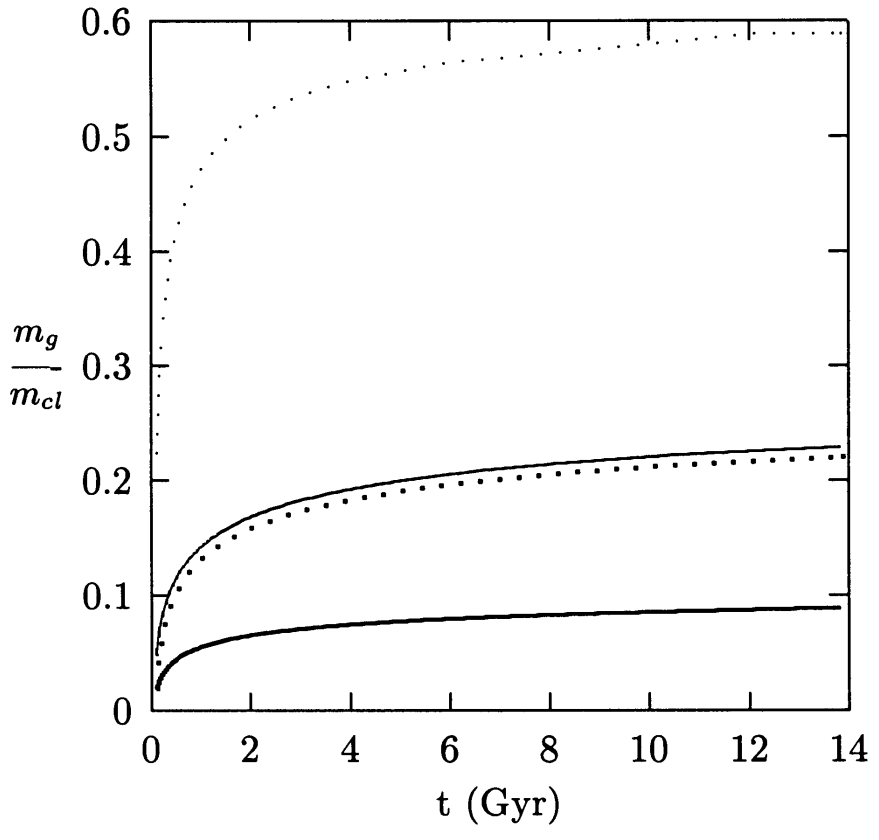

Fig. 2. Mass of gas ejected by the AGB stars, normalized to the cluster's initial mass. We have used $\alpha=2.35, m_{1}=0.1$, and $m_{\mathrm{u}}=8$ (thin solid line); $\alpha=1.5, m_{1}=0.1$, and $m_{\mathrm{u}}=8$ (thin dotted line); $\alpha=2.35, m_{1}=0.01$, and $m_{\mathrm{u}}=8$ (thick solid line); $\alpha=2.35, m_{1}=0.1$, and $m_{\mathrm{u}}=7$ (thick dotted line).

If we assume that all the gas ejected by cluster stars remains in the cluster and that the only gas-removal mechanism is by tidal sweeping when the GC crosses the galactic disk, we get a strict upper limit on the amount of gas which can be found at any time in the cluster. Even though there could have been a very large amount of gas in the cluster before its first passage through the galactic disk, this amount becomes much smaller afterwards.

\subsection{Accretion by cluster stars}

The rate of mass accretion by a star of mass $M_{\mathrm{s}}$ is given by Eq. (1), where the gas density $\rho_{\mathrm{g}}$ is determined by the amount of gas ejected by the AGB stars, but also by the ability of the cluster to retain this gas, and by the amount of gas which is accreted. We can write this equation as

$\frac{\mathrm{d} m_{\mathrm{s}}}{\mathrm{d} \tau}=2.376 \times 10^{-7} \lambda m_{\mathrm{s}}^{2} \rho v_{10}^{-3}$

where $m_{\mathrm{s}}=M_{\mathrm{s}} / M_{\odot}, \tau=t / 10^{6} \mathrm{y}, v_{10}=\left(v_{\text {rel }}^{2}+\right.$ $\left.c_{\mathrm{s}}^{2}\right)^{1 / 2} /\left(10 \mathrm{~km} \mathrm{~s}^{-1}\right)$ and $\rho=\rho_{\mathrm{g}} /\left(1 M_{\odot} / \mathrm{pc}^{3}\right)$.

The "average" gas density in the cluster can be written as

$\bar{\rho}=\frac{\left(m_{\mathrm{g}}-m_{\mathrm{a}}-m_{\mathrm{esc}}\right)}{4 \pi r_{\mathrm{g}}^{3} / 3}$

where the radius $r_{\mathrm{g}}$ of the gas reservoir is given in pc, $m_{\mathrm{a}}$ is the total mass of accreted gas, $m_{\text {esc }}$ is the mass of gas escaping from the cluster, either through a continuous wind or by tidal forces (for example when the cluster crosses the galactic disk). The actual gas density is of course a function of $r$, and we expect it to adopt some smooth profile peaking at the cluster's center and decreasing rapidly. 
Table 2. Structural parameters for some globular clusters. Unless otherwise noted, the masses $M$ and the velocity dispersions $\sigma_{\mathrm{c}}$ are from Mandushev et al. (1991), the central densities $\rho_{\mathrm{c}}$ are from Webbink (1985), the core $\left(r_{\mathrm{c}}\right)$ and tidal $\left(r_{\mathrm{t}}\right)$ radii are from the catalogue of Harris (1996), the cluster's orbital periods $(P)$ and eccentricities $(e)$ are from Dinescu et al. (1999). The escape velocities $v_{\text {esc }}$ are from Madore (1980), and $c=\log \left(r_{\mathrm{t}} / r_{\mathrm{c}}\right)$ is the concentration parameter (cc stands for "core collapse").

\begin{tabular}{|c|c|c|c|c|c|c|c|c|c|}
\hline NGC & $\begin{array}{l}M \\
\left(10^{5} M_{\odot}\right)\end{array}$ & $\begin{array}{l}\log \rho_{\mathrm{c}} \\
\left(M_{\odot} / \mathrm{pc}^{3}\right)\end{array}$ & $\begin{array}{l}r_{\mathrm{c}} \\
(\mathrm{pc})\end{array}$ & $\begin{array}{l}r_{\mathrm{t}} \\
(\mathrm{pc})\end{array}$ & $c$ & $\begin{array}{l}\sigma_{\mathrm{c}} \\
\left(\mathrm{km} \mathrm{s}^{-1}\right)\end{array}$ & $\begin{array}{l}v_{\text {esc }} \\
\left(\mathrm{km} \mathrm{s}^{-1}\right)\end{array}$ & $\begin{array}{l}P \\
\left(10^{6} \mathrm{y}\right)\end{array}$ & $e$ \\
\hline 104 (47 Tuc) & $11^{a}$ & $3.52^{d}$ & $0.2^{a}$ & 48. & 2.40 & 10.0 & 56.8 & 190-193 & 0.17 \\
\hline $5139(\omega$ Cen $)$ & $51^{b}$ & 3.35 & 3.7 & 64. & 1.24 & 16.6 & 51.2 & $120-123$ & 0.67 \\
\hline 5272 (M 3) & 6.8 & 3.86 & 1.4 & 99.8 & 1.85 & 4.8 & 34.3 & $297-321$ & 0.43 \\
\hline 5904 (M 5) & 4.6 & 4.11 & $0.90^{e}$ & $56.9^{e}$ & 1.80 & 6.5 & 34.9 & $722-995$ & 0.88 \\
\hline 6205 (M 13) & 5.8 & 3.56 & $1.3^{e}$ & $47.1^{e}$ & 1.56 & 7.86 & 30.4 & $429-526$ & 0.62 \\
\hline 6341 (M 92) & 3.9 & 4.38 & 0.54 & 34.9 & 1.81 & 6.1 & 33.1 & $201-208$ & 0.77 \\
\hline 6752 & 1.4 & 4.47 & 0.19 & 60. & $2.5(\mathrm{cc})$ & 4.9 & 31.1 & $153-156$ & 0.08 \\
\hline 7078 (M 15) & $4.9^{c}$ & $6.62^{d}$ & $0.64^{e}$ & $78.7^{e}$ & $2.1(\mathrm{cc})$ & 15.1 & 40.9 & $242-253$ & 0.31 \\
\hline
\end{tabular}

${ }^{a}$ Murphy et al. (1998); ${ }^{b}$ Meylan et al. (1995); ${ }^{c}$ Dull et al. (1997); ${ }^{d}$ Gebhardt \& Fischer (1995); ${ }^{e}$ Lehman \& Scholz (1997).

Here we assume that the gas sinks to the cluster center, forming a central reservoir of homogeneous density and of radius comparable to the core radius $r_{\mathrm{c}}$. Typical values for $r_{\mathrm{c}}$ range from 0.1 to $10 \mathrm{pc}$. If we assume that all the cluster stars have stochastic orbits, the fraction of stars in the cluster core is also the fraction of time spent by each star in the cluster core. On the other hand, stars that have sunk to the center could have smaller velocities and would tend to remain in the core for longer periods of time, accreting most of the gas (Faulkner \& Coleman 1984). Here, we use the first hypothesis, keeping in mind that some stars finding themselves preferably in the core could accrete more mass than calculated here, while others would accrete less. Using the model of King (1962), the ratio $\gamma$ of the number of stars in the core to the total number of stars in the cluster ranges between 0.3 and 0.1 approximately, for concentration parameters $c=\log \left(r_{\mathrm{t}} / r_{\mathrm{c}}\right)$ between 1 and 2 ( $r_{\mathrm{t}}$ is the tidal radius). We ignore mass segregation effects.

At a given time, a star is accreting gas at a rate which depends on its position in the cluster, its velocity with respect to the gas, and its own mass (which itself can vary with time as the star accretes or ejects gas). To get the total amount of gas which has been accreted by a given star of mass $m_{\mathrm{s}}$ at a given time $\tau$, we must integrate the mass accretion rate over time:

$m_{\mathrm{a}, \mathrm{s}}(\tau)=\gamma \int_{\tau_{1}}^{\tau} \frac{\mathrm{d} m_{\mathrm{s}}}{\mathrm{d} \tau^{\prime}} \mathrm{d} \tau^{\prime}$

where $\tau_{1}=t_{1} / 10^{6} \mathrm{y}$. We note that in Eq. (10), at any time $\tau$ we have $m_{\mathrm{s}}(\tau)=m_{\mathrm{s}, 0}+m_{\mathrm{a}, \mathrm{s}}(\tau)$ where $m_{\mathrm{s}, 0}$ is the star's initial mass. Finally, the total mass of gas accreted by the stars is obtained by integrating $m_{\mathrm{a}, \mathrm{s}}$ over the mass spectrum:

$m_{\mathrm{a}}(\tau)=\int_{m_{1}}^{m_{\mathrm{TO}}} m_{\mathrm{a}, \mathrm{s}} \frac{\mathrm{d} N}{\mathrm{~d} m} \mathrm{~d} m$.

Here, for the sake of simplicity, we have made the assumption that stars do not accrete matter after reaching the turn-off point. Indeed, those stars will instead eject matter through stellar winds. In Eq. (13), $m_{\mathrm{a}, \mathrm{s}}$ and the turnoff mass $m_{\mathrm{TO}}$ both depend on time. This is also the case for the mass spectrum $\mathrm{d} N / \mathrm{d} m$. Indeed, higher-mass stars die leaving remnants of lower masses, and some stars, especially the lower-mass stars, escape from the cluster by evaporation or through external tidal forces. The accretion process can also modify the mass spectrum if some stars accrete an appreciable fraction of their own initial mass. For simplicity, we assume that the mass spectrum remains constant with time. Since most of the gas is ejected at early times, as shown in Fig. 2, most of the accretion also takes place at those early times and we expect that during the time interval of highest accretion rate, the variation in the mass spectrum is not significant.

\section{Results}

We solve the system of Eqs. (1)-(13). We assume that the gas forms a homogeneous central reservoir of radius $r_{\mathrm{g}}=r_{\mathrm{c}}$ and that its temperature $T \approx 5000 \mathrm{~K}$ (Vandenberg \& Faulkner 1977), which gives $c_{\mathrm{s}} \approx 5.8 \mathrm{~km} \mathrm{~s}^{-1}$. We also assume that the stars have chaotic motions so that they spend about $20 \%$ of their time in the cluster core, independently of their mass and average velocity. The amount of mass accretion is proportional to the inverse cube of $v_{10}$ and $r_{\mathrm{c}}$, so that slight variations in these parameters induce large differences in the accreted mass. For the relative stellar velocities, we use the "average" value, given by the velocity dispersion in the cluster core, $\sigma_{c}$. We use the present values for the structural and dynamical parameters of the GCs, as listed in Table 2, even though those parameters could have changed as the clusters evolved chemically and dynamically. These clusters have been selected on the basis of their high escape velocities (these clusters are tightly bound and therefore more likely to retain gas) and/or because abundance anomalies have been observed for stars belonging to these clusters. The reservoir of gas is emptied each time the GC crosses the galactic 
Table 3. Results for the clusters listed in Table 2, at $t=14 \mathrm{~Gy}: m_{\mathrm{a}}$, total amount of gas that has been accreted by the cluster stars, $m_{\text {esc }}$, amount of gas which has been lost from the cluster (swept when crossing the galactic plane), $m_{\text {res }}$, the amount of residual gas in the cluster (varies between 0 just after crossing the galactic plane and its maximum value just before crossing the plane), $m_{\text {knapp }}$ the upper limit of the observed dust and ionized gas (Knapp et al. 1995, 1996), and the fraction of mass accreted by a $1 M_{\odot}$ star (last column).

\begin{tabular}{|l|l|l|l|l|l|l|}
\hline NGC & $\begin{array}{l}m_{\mathrm{a}} / m_{\mathrm{cl}, 0} \\
(\%)\end{array}$ & $\begin{array}{l}m_{\mathrm{esc}} / m_{\mathrm{cl}, 0} \\
(\%)\end{array}$ & $\begin{array}{l}m_{\mathrm{a}} / m_{\mathrm{g}} \\
(\%)\end{array}$ & $\begin{array}{l}m_{\mathrm{res}} \\
\left(M_{\odot}\right)\end{array}$ & $\begin{array}{l}m_{\mathrm{knapp}} \\
\left(M_{\odot}\right)\end{array}$ & $\begin{array}{l}m_{\mathrm{a}} / m_{\mathrm{i}},\left(m_{\mathrm{i}}=1 M_{\odot}\right) \\
(\%)\end{array}$ \\
\hline $104(47$ Tuc) & 21 & 0.2 & 98 & $<7$ & 1 & 80 \\
$5139(\omega \mathrm{Cen})$ & 0.8 & 21 & 0.4 & $<542$ & 14 & 0.1 \\
$5272($ M 3) & 5 & 16 & 24 & $<145$ & 14 & 11 \\
5904 (M 5) & 14 & 8 & 63 & $<140$ & 7 & 40 \\
6205 (M 13) & 4 & 17 & 18 & $<233$ & 7 & 8 \\
6341 (M 92) & 14 & 8 & 63 & $<33$ & 34 & 37 \\
6752 & 20 & 1 & 95 & $<1$ & 5 & 76 \\
7078 (M 15) & 4 & 18 & 16 & $<69$ & 298 & 7 \\
\hline
\end{tabular}

disk, i.e. every $P / 2$ where $P$ is the cluster's orbital period. This is the only contribution considered here for the term $m_{\text {esc }}$ appearing in Eq. (11). Other removal processes, like type I supernovae, kinetic effects of strong winds from millisecond pulsars (Spergel 1991; Freire et al. 2001) or a photoionized cluster wind driven by the UV radiation from hot stars (see Sect. 3 and Knapp 1996; Freire et al. 2001), were not included in $m_{\text {esc }}$. Any such process would decrease the efficiency of the present accretion scenario. The results presented here must therefore be considered as upper bounds.

Finally, we use $\alpha=2.35, m_{\mathrm{l}}=0.1, m_{\mathrm{u}}=8$, and $\lambda=1$ in all cases. The results at $t=14$ Gy are listed in Table 3 , where we give the total amount of gas which has been accreted by the stars, $m_{\mathrm{a}}$, the amount of gas lost from the cluster (swept when crossing the galactic plane), $m_{\mathrm{esc}}$, the maximum amount of residual gas in the cluster, $m_{\text {res }}$, and the fraction of mass accreted by a $1 M_{\odot}$ star.

Over the lifetime of the cluster, the total amount of matter injected into the GC-interstellar medium by the AGB stars is about $20 \%$ of the cluster's initial mass (see Fig. 2). In the most concentrated clusters, most of this gas is accreted by the cluster's lower-mass stars. Indeed, in 47 Tuc, NGC 6752, M 5, and M 92, more than $60 \%$ of the gas ejected by the AGB stars is accreted by the cluster stars. In those clusters, $1 M_{\odot}$ stars can accrete an appreciable fraction of their initial mass. The envelopes of those stars will reflect the composition of the intracluster medium rather than the composition of their interior, even if a physical mechanism is at work to induce mixing with the deeper layers of the star. We note here that these clusters are rich in observed stellar composition anomalies (see Sect. 4). The only cluster for which we get a very low amount of accretion is $\omega$ Cen. This is due to a combination of factors: $\omega$ Cen has a low concentration parameter compared to the other clusters we have selected. It also has a rather large core radius, and a high core velocity dispersion, as well as frequent disk crossings. On the other hand, $\omega$ Cen is a peculiar GC in many other respects and should probably not be considered as a test bed for the accretion

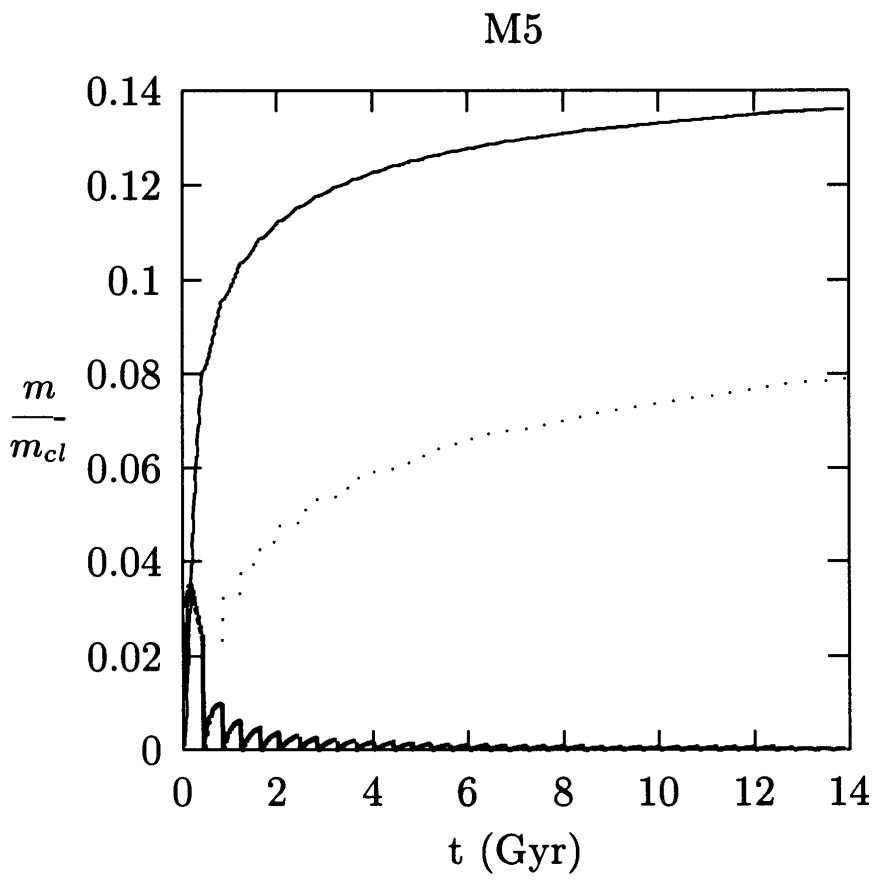

Fig. 3. Results obtained for the cluster M 5: $m_{\mathrm{a}}$ (thin solid line), $m_{\text {esc }}$ (dotted line) and $m_{\text {res }}$ (thick solid line) as a function of time.

scenario, as many studies now view $\omega$ Cen as a conglomerate of different subsystems or even as the nucleus of a dwarf galaxy (e.g., Lee et al. 1999; Hilker \& Richtler 2000; Majewski et al. 2000; Pancino et al. 2000).

The upper limits we get for the current residual intracluster gas are usually larger than the observational limits determined by Knapp et al. (1995, 1996), but of course the exact amount of gas predicted in the cluster will depend on the time elapsed since its last passage through the galactic plane. Moreover, other gas sweeping mechanisms that were not included in the present model are possibly at work, as discussed above.

The results for $m_{\mathrm{a}}, m_{\text {esc }}$ and $m_{\text {res }}$ as a function of time are shown in Fig. 3 for one particular cluster, M5. 
As expected, we find that most of the accretion takes place at early times, when the amount of gas ejected by the AGB stars is the largest. The amount of residual intracluster gas increases with time in between each passage through the galactic plane, but becomes very small at late times.

\section{Conclusions}

It was shown in this paper that accretion by low-mass GC stars of the gas ejected in the intracluster medium by moderately massive stars may be quite efficient. If enough mass is accreted, it can lead to major alteration of the stellar surface composition. This supports the EASE scenario and provides a plausible explanation for the lack of intracluster gas, and for some of the abundance anomalies observed in GC stars.

Acknowledgements. This work has been supported by the Pôle d'Attraction Interuniversitaire P4/05 (SSTC, Belgium) and by FRFC F6/15-OL-F63 (FNRS, Belgium).

\section{References}

Armosky, B. J., Sneden, C., Langer, G. E., \& Kraft, R. P. 1994, AJ, 108, 1364

Bahcall, J. N., \& Piran, T. 1983, ApJ, 267, L77

Bell, R. A., Harris, G. L. H., Hesser, J. E., \& Cannon, R. D. 1981, ApJ, 249, 637

Bell, R. A., Hesser, J. E., \& Cannon, R. D. 1983, ApJ, 269, 580

Bondi, H. 1952, MNRAS, 112, 195

Boothroyd, A. I., Sackmann, I.-J., \& Ahern, S. C. 1993, ApJ, 416,762

Briley, M. M., Smith, G. H., Bell, R. A., Oke, J. B., \& Hesser, J. E. 1992, ApJ, 387, 612

Briley, M. M., Hesser, J. E., Bell, R. A., Bolte, M., \& Smith, G. H. 1994, AJ, 108, 2183

Briley, M. M., Smith, V. V., Suntzeff, N. B., et al. 1995, Nature, 383,604

Briley, M. M., \& Cohen, J. G. 2001, AJ, 122, 242

Brown, J. A., \& Wallerstein, G. 1992, AJ, 104, 1818

Cannon, R. D., Croke, B. F. W., Bell, R. A., Hesser, J. E., \& Stathakis, R. A. 1998, MNRAS, 298, 601

Capaccioli, M., Piotto, G., \& Stiavelli, M. 1993, MNRAS, 261, 819

Charbonnel, C., Meynet, G., Maeder, A., \& Schaerer, D. 1996, A\&AS, 115, 339

Cottrell, P. L., \& Da Costa, G. S. 1981, ApJ, 245, L79

Cox, A. N. 1998, ApJ, 496, 246

Da Costa, G. S. 1997, in Fundamental Stellar Properties: The Interaction between Observation and Theory, ed. T. R. Bedding, A. J. Booth, \& J. Davis (Kluwer, Dordrecht), IAU Symp., 189, 193

D'Antona, F., Gratton, R. G., \& Chieffi, A. 1983, MSAIt, 54, 173

Dickens, R. J., \& Bell, R. A. 1976, ApJ, 207, 506

Dinescu, D. I., Girard, T. M., \& van Altena, W. F. 1999, AJ, 117,1792

Dull, J. D., Cohn, H. N., Lugger, P. M., et al. 1997, ApJ, 481, 267

Eggen, O. J., Lynden-Bell, D., \& Sandage, A. R. 1962, ApJ, 136,748
Faulkner, D. J., \& Freeman, K. C. 1977, ApJ, 211, 77

Faulkner, D. J. 1984, MNRAS, 206, 109

Faulkner, D. J., \& Coleman, C. S. 1984, MNRAS, 206, 121

Freeman, K. C. 1985, in Dynamics of Star Clusters, ed. J. Goodman, \& P. Hut (Reidel, Dordrecht), IAU Symp., 113, 33

Freire, P. C., Kramer, M., Lyne, A. G., et al. 2001, ApJ, 557, L105

Gebhardt, K., \& Fischer, P. 1995, AJ, 109, 209

Goriely, S., \& Mowlavi, N. 2000, A\&A, 362, 599

Gratton, R. L., Quarta, M. L., \& Ortolani, S. 1986, A\&A, 169, 208

Gratton, R. G., Bonifacio, P., Bragaglia, A., et al. 2001, A\&A, 369,87

Grillmair, C. J., Freeman, K. C., Irwin, M., \& Quinn, P. J. 1995, AJ, 109, 2553

Grundhal, F., Vandenberg, D., \& Andersen, M. I. 1998, ApJ, 500, L179

Hanson, R. B., Sneden, C., Kraft, R. P., \& Fulbright, J. 1998, AJ, 116, 1286

Harris, W. 1996, AJ, 112, 1487

Hilker, M., \& Richtler, T. 2000, A\&A, 362, 895

Jehin, E., Magain, P., Neuforge, C., Noels, A., \& Thoul, A. A. 1998, A\&A, 330, L33

Jehin, E., Magain, P., Neuforge, C., et al. 1999, A\&A, 341, 241

Jorissen, A., Van Eck, S., Mayor, M., \& Udry, S. 1998, A\&A, 332,877

Jorissen, A. 1999, in Asymptotic Giant Branch Stars, ed. T. Le Bertre, A. Lèbre, \& C. Waelkens, IAU Symp., 191, ASP Conf. Ser., 437

Jorissen, A., \& Meylan, G. 2001, submitted

Jurcsik, J. 1998, ApJ, 506, L113

King, I. 1962, AJ, 67, 471

King, J. R., Stephens, A., Boesgaard, A. M., \& Deliyannis, C. P. 1998, AJ, 115, 666

Knapp, G. R., Gunn, J. E., \& Connolly, A. J. 1995, ApJ, 448, 195

Knapp, G. R., Gunn, J. E., Bowers, P. F., \& Vasquez Poritz, J. F. 1996, ApJ, 462, 231

Kraft, R. P. 1994, PASP, 106, 553

Lee, Y.-W., Joo, J.-M., Sohn, Y.-J., et al. 1999, Nature, 402, 55

Lehman, I., \& Scholz, R.-D. 1997, A\&A, 320, 776

Leon, S., Meylan, G., \& Combes, F. 2000, A\&A, 359, 907

Lloyd Evans, T. 1977, MNRAS, 181, 591

Lloyd Evans, T. 1983, MNRAS, 204, 975

Lloyd Evans, T. 1986, South African Astron. Obs. Circ., 10, 1

Madore, B. 1980, in Globular Clusters, ed. D. Hanes, \& B. Madore (Cambridge University Press)

Majewski, S. R., Munn, J. A., \& Hawley, S. L. 1996, ApJ, 459, L73

Majewski, S. R., Patterson, R. J., Dinescu, D. I., et al. 2000, in The Galactic Halo: From Globular Cluster to Field Stars, Proc. of the 35th Liège International Astrophysics Colloq., ed. A. Noels, P. Magain, D. Caro, et al., Université de Liège, Liège, 619

Mandushev, G., Spassova, N., \& Staneva, A. 1991, A\&A, 252, 94

Mayor, M., Imbert, M., Andersen, J., et al. 1984, A\&A, 134, 118

Mayor, M., Duquennoy, A., Udry, S., Andersen, J., \& Nordström, B. 1996, in The Origins, Evolution, and Destinies of Binary Stars in Clusters, ed. G. Milone, \& J.-C. Mermilliod (ASP, San Francisco), ASP Conf. Ser., 90, 190 
Mayor, M., Meylan, G., Udry, S., et al. 1998, AJ, 114, 1087 McClure, R. D., \& Woodsworth, A. W. 1990, ApJ, 352, 709 Meylan, G., Dubath, P., \& Mayor, M. 1991, ApJ, 383, 587 Meylan, G., Mayor, M., Duquennoy, A., \& Dubath, P. 1995, A\&A, 303, 761

Mowlavi, N. 1999, A\&A, 350, 73

Murphy, B. W., Moore, C. A., Trotter, T. E., Cohn, H. N., \& Lugger, P. M. 1998, AAS Meeting \#193

Norris, J. E., \& Freeman, K. C. 1979, ApJ, 230, L179

Norris, J. E., Freeman, K. C., Cottrell, P. L., \& Da Costa, G. S. 1981, ApJ, 244, 205

Norris, J. E., \& Da Costa, G. S. 1995, ApJ, 447, 680

North, P., Berthet, S., \& Lanz, T. 1994, A\&A, 281, 775

Odenkirchen, M., Grebel, E. K., Rockosi, C. M., et al. 2001, ApJ, 548, L165

Pancino, E., Ferraro, F. R., Bellazzini, M., Piotto, G., \& Zoccali, M. 2000, ApJ, 534, L83

Parmentier, G., Jehin, E., Magain, P., et al. 1999, A\&A, 352, 138

Parmentier, G., Jehin, E., Magain, P., Noels, A., \& Thoul, A. A. 2000a, NewAR, 44, 351

Parmentier, G., Jehin, E., Magain, P., Noels, A., \& Thoul, A. A. 2000b, A\&A, 363, 526

Pilachowski, C. A., Sneden, C., Kraft, R. P., \& Langer, G. E. 1996, AJ, 112, 545

Piotto, G., Cool, A. M., \& King, I. R. 1997, AJ, 113, 1345

Preston, G. W., Beers, T. C., \& Shectman, S. A. 1994, AJ, 108,538
Proffitt, C. R. 1989, ApJ, 338, 990

Proffitt, C. R., \& Michaud, G. 1989, ApJ, 345, 998

Roberts, M. S. 1988, in The Harlow-Shapley Symposium on Globular Cluster Systems in Galaxies, ed. J. E. Grindlay, \& A. G. Davis Philip (Dordrecht, Kluwer), IAU Symp., 126, 411

Scott, E. H., \& Rose, W. K. 1975, ApJ, 197, 147

Scott, E. H., \& Durisen, R. H. 1978, ApJ, 222, 612

Searle, L., \& Zinn, R. 1978, ApJ, 225, 357

Shetrone, M. D. 1996, AJ, 112, 1517

Smith, V. V., Cunha, K., \& Lambert, D. L. 1995, AJ, 110, 2827

Smith, G. H. 1996, PASP, 108, 176

Smith, G. H., \& Kraft, R. P. 1996, PASP, 108, 344

Sneden, C., Kraft, R. P., Shetrone, M. D., et al. 1997, AJ, 114, 1964

Spergel, D. N. 1991, Nature, 352, 221

Suntzeff, N. B., \& Smith, V. V. 1991, ApJ, 381, 160

VandenBerg, D. A., \& Faulkner, D. J. 1977, ApJ, 218, 415

VandenBerg, D. A. 1978, ApJ, 224, 394

Vanture, A. D., Wallerstein, G., \& Brown, J. A. 1994, PASP, 106,835

Ventura, P., D'Antona, F., Mazzitelli, I., \& Gratton, R. 2001, ApJ, 550, L65

Webbink, R. F. 1985, Catalogue

Weidemann, V. 2000, AA, 363, 647

Woolley, R. 1966, Royal Observatory Annals 2 (London)

Woosley, S. E., \& Weaver, T. A. 1995, ApJS, 101, 181 